\title{
Comprehensive Analysis of Ion Beam Induced Stainless Steel Surface Morphology
}

\begin{abstract}
Z.W. KOWALSKI*
Wrocław University of Technology, Wybrzeże Wyspiańskiego 27, 50-370 Wrocław, Poland

Surface morphology, one of the various physical properties modified by ion beam irradiation, is usually characterized by well known and very often investigated surface topography and surface roughness. The paper presents two additional aspects of surface morphology modification (called by the author: profile variability and surface morphology arrangement) that seem to be good complementary indicators of morphology changes. Surface micro- and nanotopographies were observed by means of scanning electron and near-field microscopes. High quality profilograph and atomic force microscope were utilised to measure main selected roughness parameters (based on the well known surface profiles). To detect any profile shape alteration during ion beam bombardment fractal analysis was used, and especially fractal dimension $D$ that can give information about a rate $p_{\mathrm{v}}$ of profile shape variability. Surface morphology arrangement, i.e. a question whether it is random or determined, was studied with the use of frequency plots resulting from harmonic analysis of profiles. Surface topographies, selected roughness parameters, fractal dimensions and frequency plots relating to stainless steel of $1 \mathrm{H} 18 \mathrm{~N} 9 \mathrm{~T}$ (made in Poland) bombarded with perpendicular $\left(\Theta=0^{\circ}\right)$ as well as very inclined $\left(\Theta=85-87^{\circ}\right)$ beam were examined. Low energy $(0.8 \mathrm{keV})$ broad argon ion beam and neutralized narrow argon or krypton ion beams (up to $6 \mathrm{keV}$ ) were used in the experiments.
\end{abstract}

PACS: $79.20 . \mathrm{Rf}$

\section{Introduction}

The term "morphology" comes from Greek and consists of two words: morphe $=$ shape and logos = learning, science, knowledge. In this connection the term "surface morphology" should mean, and usually means, knowledge about surface shape/surface sculpture (generally in micro- and nanoscale). Surface modification processes like surface polishing/smoothing [1, 2], surface roughening [3], surface nanostructuring [4], surface nanopatterning $[5,6]$ can be achieved by miscellaneous technologies [7-9] (mechanical, chemical, physical), among others with the use of ion irradiation [1-6]. The processes in question are important and should be considered in various areas of science and technology, for example: (a) in microelectronics where shrinking semiconductor dimensions cause that resist line edge roughness (LER) will be more important because roughness from the resist is transferred to the substrate with further processing steps [10] (for the patterning of sub-100 nm features, clear understanding of the origin and control of LER is extremely desirable, from a fundamental as well as a manufacturing perspective $[11,12])$, (b) in surface analysis [13] where ion beam sputtering is widely used in depth-profile analytical techniques such as, for instance, Auger electron spectroscopy (AES), Rutherford

\footnotetext{
* corresponding author; e-mail:

Zbigniew.Wojciech.kowalski@pwr.wroc.pl
}

backscattering (RBS), secondary ion mass spectroscopy (SIMS) or X-ray photoelectron spectroscopy (XPS).

Surface morphology is usually characterized by well known and very often investigated: surface topography and surface roughness. The paper presents two additional aspects of morphology modification, called by the author: profile variability and surface morphology arrangement, that seem to be good complementary indicators of morphology changes. Therefore comprehensive analysis presented here concentrates on:
a) topography,
b) roughness,
c) profile variability, and
d) morphology arrangement.

Changes of the four surface morphological properties $(\mathrm{a} \div \mathrm{d})$ induced by normal as well as very inclined ion beam irradiation of stainless steel were investigated. Broad argon as well as neutralized narrow argon or krypton ion beams were used in experiments. The main aim of the paper is to give a relatively full picture of surface morphological changes.

\section{Experimental details}

Stainless steel specimens of 1H18N9T (made in Poland) delivered as preliminary ground were worked to form disks: $15 \mathrm{~mm} \times 1.5 \mathrm{~mm}$ (diameter $\times$ thickness). 
The steel in question is rather sputter resistant (low-sputter yield) material. Ion irradiation processes at normal $\left(\Theta=0^{\circ}\right)$ as well as at very inclined $\left(\Theta=85-87^{\circ}\right)$ incidence were performed in two pieces of experimental apparatus: (a) with the glow discharge ion gun with a hollow anode [14] (at an applied voltage 5-6 kV) used as a neutralized narrow argon (anode current up to $0.4 \mathrm{~mA}$ ) and krypton (anode current up to $0.25 \mathrm{~mA}$ ) ion beam source, (b) with the ion source based on the Kaufman ion thruster concept developed by NASA, similar to that described in Ref. [15], where the specimens were bombarded by the $12 \mathrm{~cm}$ diameter argon ion beam at an applied voltage of $0.8 \mathrm{kV}$ and at the ion current density of about $0.5 \mathrm{~mA} / \mathrm{cm}^{2}$. During normal bombardment the specimens were rotated.

Surface micro- and nanotopographies were observed by means of scanning electron and near-field microscopes. To this end Hitachi S-570 or Jeol JSM-5800LV (low vacuum) scanning electron microscopes as well as calibrated homebuilt contact mode atomic force microscope were utilized. Surface roughness, described by three selected parameters, was measured before ion irradiation and after each ion bombardment process, using a high quality profilograph — Rank Taylor Hobson's Talysurf or the AFM. The above-mentioned parameters, i.e. $R_{\mathrm{a}}$ arithmetical mean deviation of the profile (it gives information about vertical features of roughness profile), $S$ - mean spacing of local peaks of the profile and $S_{\mathrm{m}}$ mean spacing of the profile irregularities (they give information about horizontal features of roughness profile) are normalized and often used. To measure those parameters properly, surface profiles must be known well. A significant drawback of this measuring method is that one can find profiles with the same values of roughness parameters but with different profile shapes - that could mean various physical properties of the surface in question. To detect any profile shape alteration induced by ion beam irradiation fractal analysis was used, and especially fractal dimension $D$ that can give information about a rate $p_{\mathrm{v}}$ of profile variability (profile shape variability). For example, if $D=$ const after the bombardment process, the shape does not depend on time of ion irradiation (does not change). Surface morphology arrangement, i.e. a question whether it is undefined (random) or defined (determined), was studied with the use of frequency plots resulting from harmonic analysis of profiles.

\section{Results and discussion}

\subsection{Roughness and topography}

Stainless steel $R_{\mathrm{a}}$ changes, examined by the profilograph, are shown in Figs. 1-3 (the points in all presented figures are mean values of many measurements and the smooth curves are polynomial or exponential fits). For normal ion bombardment (see Fig. 1) the curves increase substantially with time $t$ but differences between $R_{\mathrm{a}}$ values for certain bombardment duration are visible [16]. They are lower for various argon ion energies (0.8 keV

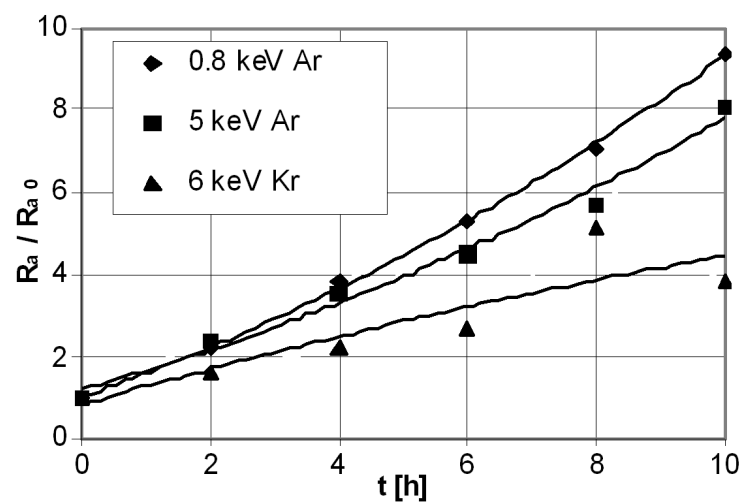

Fig. 1. Normal $\left(\Theta=0^{\circ}\right)$ argon ion beam induced changes of stainless steel surface relative roughness $R_{\mathrm{a}} / R_{\mathrm{a} 0}$ (initial roughness: $R_{\mathrm{a} 0 / 0.8 \mathrm{keV}}=73.5 \mathrm{~nm}$, $\left.R_{\mathrm{a} 0 / 5 \mathrm{keV}}=84.5 \mathrm{~nm}, R_{\mathrm{a} 0 / 6 \mathrm{keV}}=123.3 \mathrm{~nm}\right)[16]$.

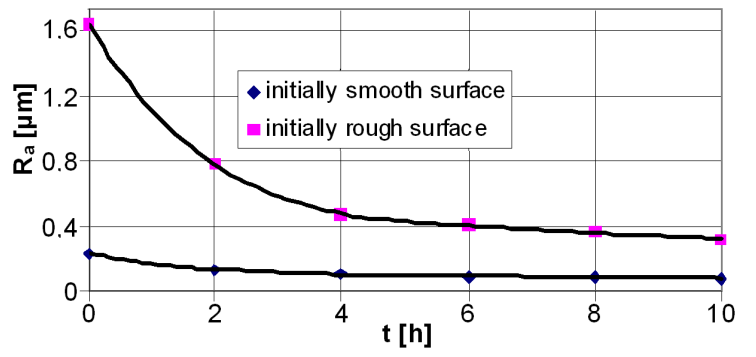

Fig. 2. The influence of initial surface roughness $R_{\mathrm{a} 0}$ on vertical roughness parameter $R_{\mathrm{a}}$ of stainless steel due to neutralized narrow argon ion beam bombardment at $\Theta=87^{\circ}[18]$.

and $5 \mathrm{keV}$ ) and bigger for various kinds of ions (Ar, $\mathrm{Kr}$ ) with very similar energies $(5 \mathrm{keV}, 6 \mathrm{keV})$. The main reason of the differences observed for argon irradiation is an initial surface roughness $R_{\mathrm{a} 0}$, i.e the roughness of untreated surface (before ion irradiation process). The influence of initial surface roughness on ion induced surface morphology is quite important and must be taken into account — see Fig. 2 and e.g. Ref. [17]. Bigger differences in the case of krypton and argon irradiation are

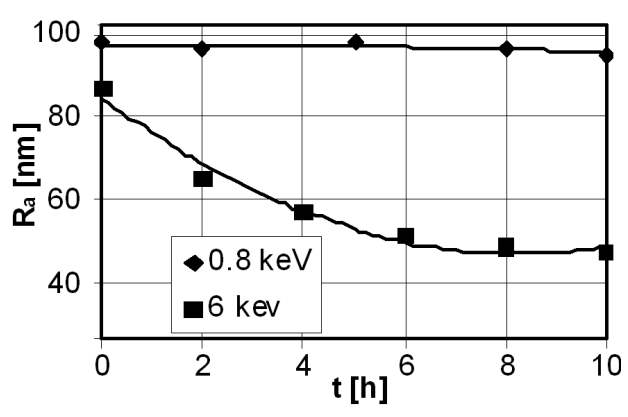

Fig. 3. Steel surface roughness $R_{\mathrm{a}}$ vs. time of inclined argon ion irradiation [19]. 


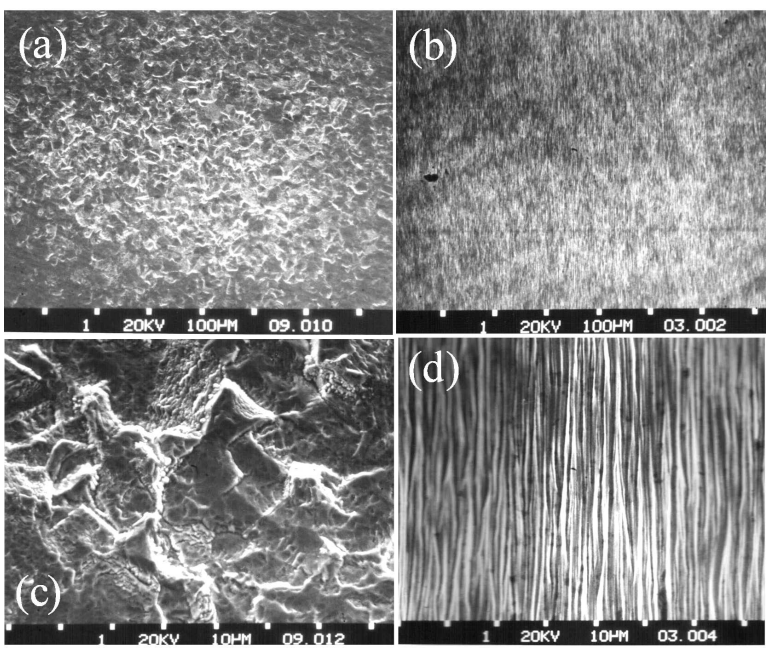

Fig. 4. Ex situ SEM images of surface topographies induced on stainless steel surface with the use of neutralized argon ion beam: (a) and (c) perpendicular $\left(\Theta=0^{\circ}\right),(\mathrm{b})$ and $(\mathrm{d})$ very inclined $\left(\Theta=87^{\circ}\right)$.

due to smaller ion dose used in the experiments. Quasi-tangential bombardment $\left(\Theta=85-87^{\circ}\right)$ of steel surface results in diminishing of $R_{\mathrm{a}}$ values [19] but with various dynamics of polishing processes for $0.8 \mathrm{keV}$ and $6 \mathrm{keV}$ (see Fig. 3). SEM images of stainless steel surfaces after normal and narrow neutralized argon ion beam irradiation are shown in Fig. 4.
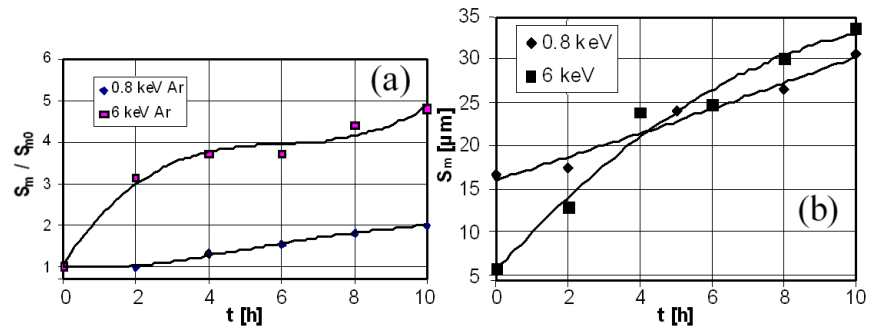

Fig. 5. Changes of horizontal roughness parameters due to argon ion beam bombardment [16, 19]: (a) relative spacing $S_{\mathrm{m}} / S_{\mathrm{m} 0}$ for normal irradiation $\left(S_{\mathrm{m} 0 / 0.8 \mathrm{keV}}=19.5 \mu \mathrm{m}, S_{\mathrm{m} 0 / 6 \mathrm{keV}}=6 \mu \mathrm{m}\right)$, (b) mean spacing of profile irregularities $S_{\mathrm{m}}$ for very inclined bombardment.

Together with modification of vertical roughness the horizontal parameters $S_{\mathrm{m}}$ and $S$ also change. Figure 5 shows the influence of bombardment duration on $S_{\mathrm{m}}$ and $S_{\mathrm{m}} / S_{\mathrm{m} 0}$ for the material investigated $[16,19]$. The results presented in Fig. 5a and b mean widening of convex topographical forms through eliminating of small dimension features by more extensive elements, development of some topographical forms "at the cost" of the others, independently of ion beam inclination. That was proved by the SEM studies [20-22] and can be observed in the micrographs presented in Figs. 6 and 7. Widening of topographical elements resulting from ion irradiation, as

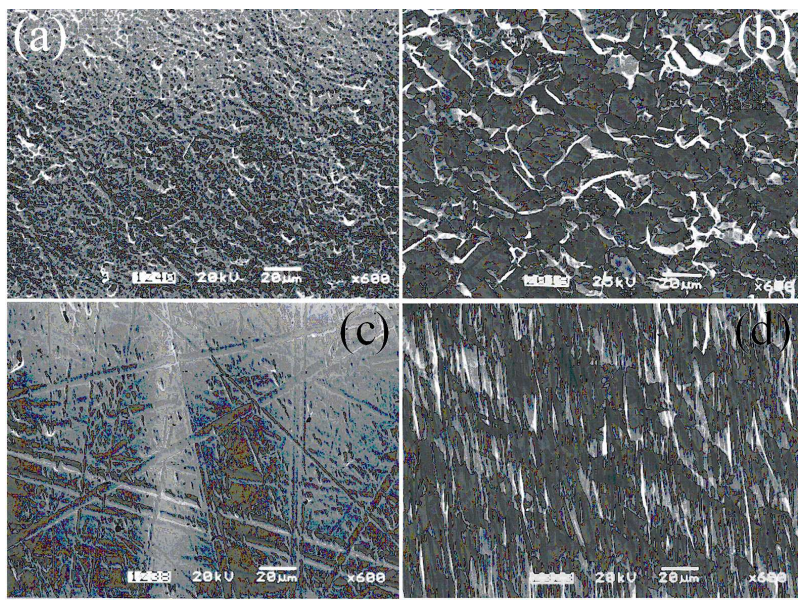

Fig. 6. Ex situ scanning electron microscopy images of steel surface topography after broad argon ion beam bombardment [20,21]: (a) $\Theta=0^{\circ}, 1 \mathrm{~h}$, (b) $\Theta=0^{\circ}$, $6 \mathrm{~h}$, (c) $\Theta=85^{\circ}, 2 \mathrm{~h}$, (d) $\Theta=85^{\circ}, 25 \mathrm{~h}$.

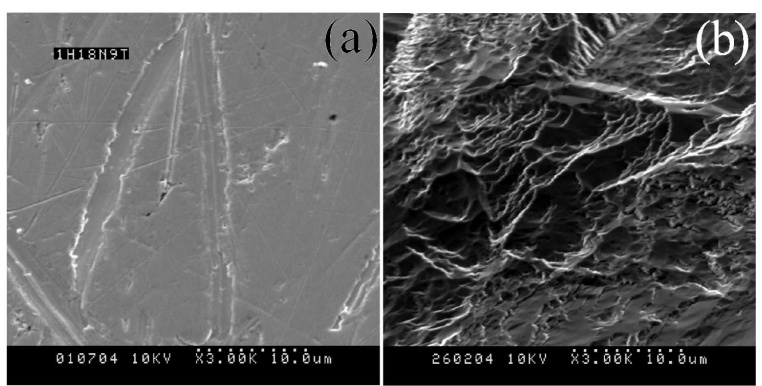

Fig. 7. Ex situ scanning electron microscopy images of steel surface topography after normal krypton neutralized ion beam irradiation [22]: (a) $0 \mathrm{~h}$, (b) $10 \mathrm{~h}$.

shown in Fig. 5a and b, is in good agreement with the ex situ SEM studies.

All the roughness parameters were measured over a range of a conventionally determined and normalized elementary segment $l$. It is worth noting here that values of the parameters in question depend on the above-

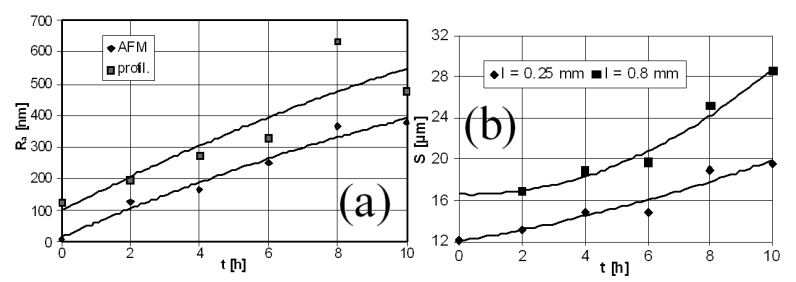

Fig. 8. "Scaling effects" due to various lengths of element $l$ (scale) used in surface roughness measurements of stainless steel irradiated with $6 \mathrm{keV}$ neutralized krypton ion beam. In that case lengths of element $l$ : (a) $0.25 \mathrm{~mm}$ for profilograph and $0.008 \mathrm{~mm}$ for AFM, (b) $0.8 \mathrm{~mm}$ and $0.25 \mathrm{~mm}$ for profilograph. 
-mentioned segment as shown in Fig. 8a (parameter $R_{\mathrm{a}}$ ) and Fig. 8b (parameter $S$ ). If the length of element $l$ (scale) was reduced: (a) from $0.25 \mathrm{~mm} /$ profilograph to $0.008 \mathrm{~mm} / \mathrm{AFM}$, and (b) from $0.8 \mathrm{~mm} /$ profilograph to $0.25 \mathrm{~mm} /$ profilograph, the surfaces appeared to be smoother and horizontal features seem to be narrower (see for example [16]). That "scaling effect" is very important and must be taken into account during roughness measurements.

\subsection{Profile variability}

To detect any profile alteration, induced by ion bombardment, the fractal dimension $D$ was used that can give information about the rate of profile shape variability

$$
p_{\mathrm{v}}=\frac{D}{D_{0}}=\frac{D(t)}{D(t=0)},
$$

where $D$ is the fractal dimension calculated after time $t$ of ion irradiation, $D_{0}$ is the dimension calculated for untreated (initial) surface.

To study the rate in question the Fourier method [23] of fractal analysis of surface roughness profiles was applied (see for instance [21, 24]). In the method profile function $f(x)$, i.e. profilogram, is approximated by

$$
f(x)=\sum_{k}^{l} a_{k} \sin (2 \pi k x),
$$

where $a_{k}$ is the amplitude of $k$ harmonic.

Drawing the function $\log a_{k}^{2}=\log (2 \pi k x)$, one can obtain a lot of points that can be approximated by the straight line: $y=m x+b$. Fractal dimension is proportional to the inclination $m$ of the line in question. It was calculated with the help of Benoit 1.3 program and determined as $D=(5+m) / 2$.

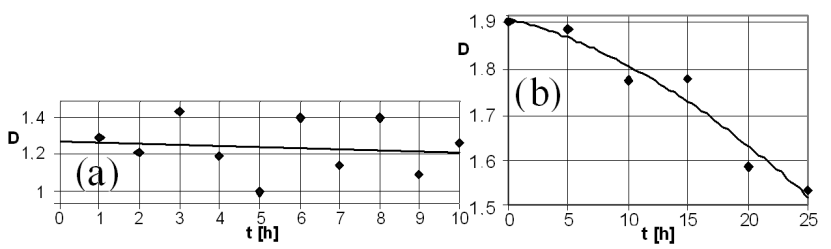

Fig. 9. Changes of fractal dimension $D$ as a function of broad argon ion beam bombardment duration of stainless steel surface: (a) $\Theta=0^{\circ}$, (b) $\Theta=85^{\circ}$.

TABLE

Rate of profile shape variability $p_{\mathrm{v}}$ vs. time $t$ of argon broad ion beam bombardment of stainless steel surface for normal and very inclined irradiation, calculated for smooth curves presented in Fig. 9a and b.

\begin{tabular}{c|c|c|c|c|c|c|c}
\hline \hline$t[\mathrm{~h}]$ & 0 & 2 & 4 & 6 & 8 & 10 & $\Delta p_{\mathrm{v}}$ \\
\hline$p_{\mathrm{v}}\left(\Theta=0^{\circ}\right)$ & 1 & 0.994 & 0.986 & 0.974 & 0.963 & 0.959 & $\approx 4$ \\
\hline \hline$t[\mathrm{~h}]$ & 0 & 5 & 10 & 15 & 20 & 25 & $\Delta p_{\mathrm{v}}$ \\
\hline$p_{\mathrm{v}}\left(\Theta=85^{\circ}\right)$ & 1 & 0.979 & 0.942 & 0.906 & 0.853 & 0.801 & $\approx 20$ \\
\hline
\end{tabular}

$\Delta p_{\mathrm{v}}$ - deviation of the rate from mean value of $p_{\mathrm{v}}$ (in [\%])
Figure 9 shows the fractal dimension $D$ as a function of bombardment duration, calculated for stainless steel irradiated with the use of perpendicular and very inclined $\left(\Theta=85^{\circ}\right)$ broad argon ion beam. Mean value of fractal dimension (smooth curve) seems to be nearly constant during normal $\left(\Theta=0^{\circ}\right)$ bombardment process (Fig. 9a). It means that the shape of the steel surface profile depends not much on time of ion irradiation. The opposite results can be observed for inclined bombardment (Fig. 9b) where fractal dimension decreased distinctly. Table presents the influence of bombardment duration on the rate $p_{\mathrm{v}}$ of profile shape variability (calculated for smooth curves in Fig. 9). It gives information whether initial (i.e. before irradiation) surface roughness profile shape changes or does not change due to the ion beam treatment.

\subsection{Surface morphology arrangement}

To characterize the fourth aspect, i.e. surface morphology arrangement induced by ion beam irradiation, harmonic analysis borrowed from electrical engineering, treating a surface roughness profile as an electrical signal, was used. In the analysis in question [23] every periodical signal can be presented as the infinite sum of harmonic functions

$$
f(t)=\sum_{k=0}^{\infty} a_{k} \sin \left(2 \pi k t-\theta_{k}\right),
$$

where $\theta_{k}$ is the phase of $k$-harmonic.

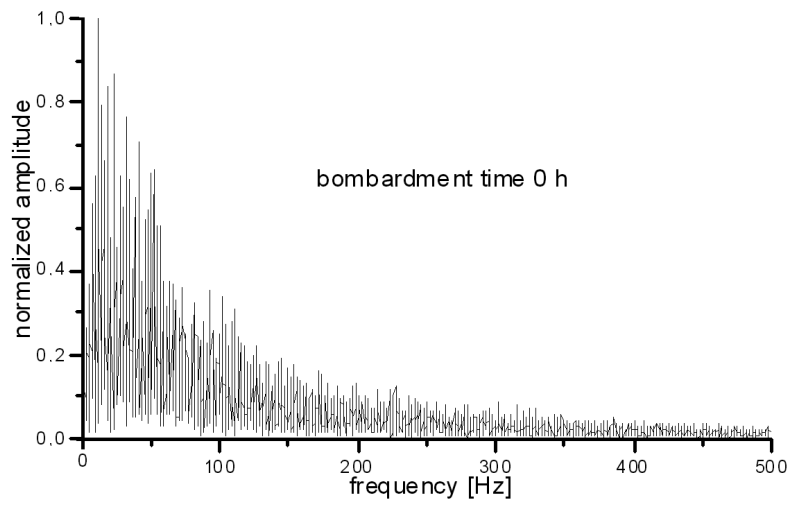

Fig. 10. Typical frequency plot for broad argon ion beam irradiation.

Frequency plots (as the result of harmonic analysis) can give information about surface morphology arrangement, i.e. whether it is random or determined. The wider frequency band, the more random surface morphology and, on the contrary, the narrower frequency band, the more determined surface morphology. The typical frequency plot, here for untreated surface, is shown in Fig. 10. Amplitudes of all harmonic components were normalized in relation to the maximum amplitude. The frequency band for every surface roughness profile was defined until the amplitudes of successive harmonic components were equal to 0.1 maximum amplitude. With 
the increase of steel surface bombardment duration diminishing of frequency band can be observed, for example: (a) for untreated surface this is about $400 \mathrm{~Hz}$, (b) for $5 \mathrm{~h}$ of very inclined argon ion beam bombardment this is about $150 \mathrm{~Hz}$, and (c) for $15 \mathrm{~h}$ of irradiation this is about $100 \mathrm{~Hz}$. It means that the surface morphology is more determined ("less random") due to oblique bombardment. The effect in question is less visible for normal ion beam treatment (see e.g. titanium in [16]).

\section{Conclusion}

The paper tries to present a relatively full picture of surface morphology changes induced by $0.8-6 \mathrm{keV}$ argon and krypton ion beam irradiation of $1 \mathrm{H} 18 \mathrm{~N} 9 \mathrm{~T}$ stainless steel (made in Poland) taking into consideration four aspects of the morphology modification in question. The two of them are known and often studied: (a) surface topography, and (b) surface roughness. Two further aspects are new and seem to be important: (c) profile variability, and (d) surface morphology arrangement.

Scanning electron and atomic force microscopes as well as high quality profilograph were applied to examine ion beam induced surface morphology modification. Such investigative procedure allowed to study the changes of roughness and topography in various scales. The results of measurements and calculations, obtained for various lengths of elementary segment $l$, showed "scaling effects": generally similar changes of roughness parameters but not in the same scale. Argon and krypton ion beam induced deepening (normal bombardment), smoothing (inclined irradiation) and widening (normal as well as inclined irradiation) of topographical elements observed in roughness results of experiments are in good agreement with the scanning electron microscopy studies. Profile variability was determined with the use of the rate $p_{\mathrm{v}}$. To detect the rate $p_{\mathrm{v}}$ of profile shape variability fractal dimension $D$ has to be calculated. The parameter in question $\left(p_{\mathrm{v}}\right)$ was nearly constant during normal broad argon ion beam bombardment of steel. This means that the shape of surface profile depends not much on time of ion irradiation. The opposite results were observed for inclined bombardment where the rate decreased distinctly. The frequency plots were used to give information about surface morphology arrangement - the wider frequency band, the more random surface morphology. The results of experiments and calculations have shown that with the increase of oblique ion beam bombardment duration diminishing of frequency band can be observed. It means that the sputtered stainless steel surface morphology is more determined ("less random").

It seems that comprehensive analysis of surface topography, surface roughness, profile variability, and surface morphology arrangement can give quite a good (precise) picture of surface modification induced by various technologies (e.g. mechanical treatment, chemical etching, and others), especially by ion beam irradiation.

\section{References}

[1] F. Frost, R. Fechner, B. Ziberi, J. Völlner, D. Flamm, A. Schindler, J. Phys., Condens. Matter 21, 4026 (2009).

[2] C. Hofer, S. Aberman, C. Teickert, T. Bobek, H. Kurz, K. Lyutovich, E. Kasper, Nucl. Instrum. Methods Phys. Res. B 216, 178 (2004).

[3] N.V. Medhekar, W.L. Chan, V.B. Shenoy, E. Chason, J. Phys., Condens. Matter 21, 4021 (2009).

[4] Debabrata Ghose, J. Phys., Condens. Matter 21, 4001 (2009).

[5] R. Cuerno, L. Vázquez, R. Gago, M. Castro, J. Phys., Condens. Matter 21, 301 (2009).

[6] J.A. Sánchez-García, R. Gago, R. Caillard, A. Redondo-Cubero, J.A. Martin-Gago, F.J. Palomares, M. Fernández, L. Vázquez, J. Phys., Condens. Matter 21, 4009 (2009).

[7] B. Sikora, Ph.D. Thesis, Karol Marcinkowski Medical University in Poznań, Poznań 2005.

[8] K. Kupiec, P. Konieczka, J. Namieśnik, Ecolog. Chem. Eng. S T.14, S4, 217 (2007).

[9] J. Radziejewska, W. Kalita, B. Nowicki, Przeglad Mechaniczny LXV, 17 (2006).

[10] G.W. Reynolds, J.W. Taylor, J. Vac. Sci. Technol. B 16, 2723 (1999).

[11] D.L. Goldfarb, A.P. Mahorowala, G.M. Gallatin, K.E. Petrillo, K. Temple, M. Angelopoulos, S. Rasgon, H.H. Sawin, S.D. Allen, M.C. Lawson, R.W. Kwong, J. Vac. Sci. Technol. B 22, 647 (2004).

[12] S. Engelmann, R.L. Bruce, T. Kwon, R. Phaneuf, G.S. Oehrlein, Y.C. Bae, C. Andes, D. Graves, D. Nest, E.A. Hudson, P. Lazzeri, E. Iacob, M. Anderle, J. Vac. Sci. Technol. B 25, 1353 (2007).

[13] R. Loesing, G.M. Guryanov, J.L. Hunter, D.P. Griffis, J. Vac. Sci. Technol. 18, 509 (2000).

[14] J. Wilk, J. Martan, Z.W. Kowalski, in: Proc. Sixth Sci. Conf. on Electronic Technology, ELTE'97, Krynica (Poland), Vol. 2, AGH Kraków 1997, p. 478.

[15] S. Łasisz, Z. Radzimski, I. Rangelow, Direktabb Oberfl 17, 69 (1984).

[16] Z.W. Kowalski, J. Wilk, J. Martan, Vacuum 83, 208 (2009).

[17] P. Karmakar, S.A. Mollick, D. Ghose, A. Chakrabarti, Appl. Phys. Lett. 93, 103102 (2008).

[18] Z.W. Kowalski, J. Wilk, Mater. Sci.-Poland 26, 279 (2008).

[19] Z.W. Kowalski, J. Wilk, Elektronika 50, 74 (2009).

[20] G. Przybylski, J. Martan, Z.W. Kowalski, Vacuum 70, 255 (2003).

[21] J. Martan, R. Tabaka, G. Przybylski, Z.W. Kowalski, Vacuum 78, 217 (2005).

[22] J. Bałamucki, P. Czarnecki, T. Gotszalk, A. Marendziak, I. Rangelow, J. Wilk, Z.W. Kowalski, Phys. Chem. Solid State 7, 577 (2006).

[23] J.C. Russ, Fractal Surfaces, Plenum Press, New York 1994.

[24] J. Martan, Z.W. Kowalski, Elektronika 42, 16 (2001). 